\title{
ON THE NUMERICAL SOLUTION OF THE DIRICHLET PROBLEM FOR LAPLACE'S DIFFERENCE EQUATION*
}

\author{
By \\ J. B. DIAZ AND R. C. ROBERTS \\ Institute for Fluid Dynamics and Applied Mathematics, University of Maryland
}

1. Introduction. The present note is concerned with the proof of the convergence of three iterative methods for the numerical solution of the difference equation boundary value problem which is analogous to the classical Dirichlet problem for Laplace's differential equation. These three iterative methods for the Dirichlet difference equation problem bear a marked resemblance to, and are patterned after, methods of solution for the Dirichlet differential equation problem, and may be briefly characterized as follows: (a) method I is the precise analogue of Poincaré's [1] "methode de balayage", (b) method II is the precise analogue of the extension of Poincaré method due to Kellogg [2], (c) method III is based on a certain connection between Laplace's differential equation and the heat equation (intuitively put, "the steady state temperature is a harmonic function")'

Several convergence proofs for the Dirichlet difference boundary value problem have been based on Liebmann's [3] original proof (see P. Frank and R. von Mises [4], and I. G. Petrowsky [5]) which involves a definite ordering of the points of the domain. It follows from the convergence proof given below for method III that this preliminary ordering of the points of the domain is unnecessary. R. Courant [6], has given a convergence proof for method I for the Dirichlet difference boundary value problem employing a variational method which is the direct analogue of the classical Dirichlet's principle for Dirichlet's differential boundary value problem. In spite of the inherent interest and symmetry of the proofs in methods I and II described below, it should be remarked that the variational method of proof (minimization of a quadratic sum) proposed by Courant possesses a certain advantage in that it may be applied to other "elliptic" difference equation boundary value problems, since it does not require for its application the maximum-minimum property of the solutions of the difference equation, i.e. that "the maximum and the minimum of a solution of the difference equation occurs on the boundary". In the Laplace case, both the differential and the difference equations possess the maximum-minimum property. However, it is easy to give examples of elliptic differential equations (for which the maximum-minimum principle holds) e. g. $U_{x x}+U_{x y}+U_{y y}=0$, which have the property that the seemingly most naturally associated difference equation does not possess the maximum-minimum property. A convergence proof for method III, different from the one given here, has been given by Feller [7].

Although only the two dimensional case will be considered here explicitly, it is clear that the same arguments are valid in a finite number of dimensions.

2. Convergence proofs. The boundary value problem will be formulated first. Consider a finite set $S$ of points $(m, n)$ in the $x y$-plane, where $m$ and $n$ are integers (i.e., $S$ is "a net of equally space points in the plane, where for convenience the spacing has

${ }^{*}$ Received May 4, 1951. This work was carried out under Contract N7onr-39705, sponsored by the Office of Naval Research.

${ }^{1}$ Numbers in brackets refer to the bibliography at the end of the paper. 
been taken as unity"). The points of the set $S$ are divided into two mutually exclusive subsets of points, the subset $D$ consisting of interior points of $S$ and the subset $C$ of boundary points of $S$. A point $(m, n)$ of $S$ is said to be an interior point of $S$ provided that its "four neighbors" $(m \pm 1, n),(m, n \pm 1)$ also belong to $S$. A point $(m, n)$ of $S$ is said to be a boundary point of $S$ provided that at least one of its four neighbors $(m \pm 1, n),(m, n \pm 1)$ does not belong to $S$. It will be supposed in what follows that every point of the boundary $C$ has at least one neighbor in $D$ (this is clearly no restriction, as far as the difference equation problem is concerned, since only "isolated" points of the set $S$ and "unessential" boundary points of $S$ are excluded).

The Dirichlet difference boundary value problem for the finite set $S=D+C$ consists in finding a real valued function $u$, defined on $D+C$, assuming prescribed values on the boundary $C$ and which satisfies Laplace's difference equation

$$
4 u(m, n)=u(m+1, n)+u(m-1, n)+u(m, n+1)+u(m, n-1),
$$

for each point $(m, n)$ in the interior $D$.

This boundary value problem, which is analogous to the classical Dirichlet problem, is well known to possess one and only one solution. This is readily seen as follows. The difference equation (1) amounts to a system of $d$ linear equations in the $d$ unknown values of $u$ at the points of $D$, supposed to be $d$ in number. This system of linear equations is homogeneous if and only if the prescribed values of $u$ on $C$ are all zero (recall that it was assumed that every point of $C$ has at least one neighbor in $D$ ). But it is known from algebra that a system of $d$ linear equations in $d$ unknowns will have a unique solution if and only if the corresponding homogeneous system has only the trivial, identically zero solution. That the homogeneous system has only the zero solution follows immediately once it is shown that the maximum and minimum values of a function $u$, defined on $D+C$, and satisfying (1) in $D$, must occur on the boundary $C$. Suppose, on the contrary, that the maximum value $M$ of $u$ occurs at a point $(m, n)$ of $D$, but not on the boundary $C$. Then from (1) it follows that the value of $u$ at the four points ( $m \pm 1, n),(m, n \pm 1)$ must also be $M$ and hence these four points must all be in the interior, $D$. By induction, it follows that the infinite set of points $(m+j, n), j=0,1,2$, ... all belong to $D$, which contradicts the initial assumption that $D$ is a finite set of points. Thus the maximum value $M$ must occur at a point of $C$. Since a minimum of the function $u$ is a maximum of the function $-u$, and $-u$ also satisfies (1), it follows that the minimum of $u$ on $D+C$ also occurs on $C$.

\section{Method $I$.}

Consider the convergence of method I mentioned in the introduction. Denote by $f$ the given real valued function, defined on $C$, which is the prescribed boundary value of $u$ on $C$, and let the "initial function" $G$ be a superharmonic function defined on $D+C$ and which coincides with the given function $f$ on the boundary $C$. That is

$$
4 G(m, n) \geqq G(m+1, n)+G(m-1, n)+G(m, n+1)+G(m, n-1),
$$

for each $(m, n)$ in $D$, and

$$
G(m, n)=f(m, n),
$$

for each $(m, n)$ in $C$. The restriction that the initial function $G$ be superharmonic is not essential, and will be removed later. 
Let the points of $D$ be arranged in a sequence $P_{1}, P_{2}, P_{3}, \cdots$ in such a way that each point of $D$ occurs infinitely many times in the sequence. For convenience, the sequence may be chosen by moving from point to point of $D$ in some definite geometrical pattern, but this is not necessary.

A sequence of functions is now defined on $D+C$ by the following procedure. First, let $w_{0} \equiv G$, and define the sequence of functions $w_{0}, w_{1}, w_{2}, \cdots$ successively as follows:

First step: $w_{0} \equiv G$, on $D+C$;

Second step: $w_{1}$ is harmonic at $P_{1}$, $w_{1}=w_{0}$ on $D+C-P_{1}$;

Third step: $w_{2}$ is harmonic at $P_{2}$, $w_{2}=w_{1}$ on $D+C-P_{2}$;

$(p+1)$ st. step: $w_{p}$ is harmonic at $P_{p}$,

$$
w_{p}=w_{p-1} \text { on } D+C-P_{p} ;
$$

etc. In other words, one moves from point to point in $D$ changing the value of the functions at each point so that equation (1) holds.

It is easily seen that each function $w_{p}$ is superharmonic in $D+C$. Consider $w_{1}$, and let $P_{1}=(m, n) . w_{1}$ is certainly superharmonic at all points of $D+C$, save perhaps at the five points $(m, n),(m \pm 1, n),(m, n \pm 1)$. But at $(m, n)$ the equality sign holds in equation (2), with $G$ replaced by $w_{1}$, by the definition of $w_{1}(m, n)$, while at the four points $(m \pm 1, n),(m, n \pm 1)$, equation (2), with $G$ replaced by $w_{1}$, holds a fortiori, since the right hand sum does not increase if $G$ is replaced by $w_{1}$. By induction, $w_{p}$ is superharmonic for each $p$. Further, the sequence of functions $w_{0}, w_{1}, w_{2}, \cdots$ is monotonically non-increasing on $D+C$, by the way in which the sequence was constructed. Finally, each function $w_{p}$ is bounded below by the minimum value of $G$ on $D+C$.

This last statement follows immediately from the fact that the minimum value of a function superharmonic in $D+C$ must occur at a point of $C$ (this may be seen by an argument analogous to that used above in proving the maximum-minimum property of solutions of (1)). Since

$$
w_{p}(m, n)=G(m, n)=f(m, n),
$$

for $(m, n)$ in $C$ and any $p$, it follows that for any $p$

$$
w_{p}(m, n) \geqq \min _{c} G=\min _{C} f,
$$

for any $(m, n)$ in $D$; and this, together with

$$
w_{1}(m, n) \geqq w_{2}(m, n) \geqq \cdots \geqq w_{p}(m, n) \geqq \cdots
$$

assures the convergence of the sequence $w_{p}$ at each point of $D$. It only remains to show that the limit function

$$
\lim _{p \rightarrow \infty} w_{p}(m, n),
$$

$(m, n)$ in $D+C$, is the solution of the boundary value problem. 
There is no question about the fact that the limit function coincides with $f$ on $C$, since each $w_{p}$ does, it is only necessary to show that the limit function satisfies the partial difference equation (1) in $D$. Consider a point $(m, n)$ of $D$. Since $(m, n)$ occurs infinitely many times in the sequence $P_{1}, P_{2}, P_{3}, \cdots$ it follows that there is an infinite sequence of integers, $k_{p}$, such that

$$
(m, n)=P_{k_{p}},
$$

for $p=1,2,3, \cdots$. Consequently, there is a subsequence of functions $w_{k_{1}}, w_{k_{2}}, \cdots$, $w_{k_{p}}, \cdots$ of the sequence $w_{1}, w_{2}, \cdots, w_{p}, \cdots$ such that each function $w_{k_{p}}$ of the subsequence is harmonic at $(m, n)$, that is

$$
4 w_{k_{p}}(m, n)=w_{k_{p}}(m+1, n)+w_{k_{p}}(m-1, n)+w_{k_{p}}(m, n+1)+w_{k_{p}}(m, n-1) .
$$

Since

$$
\lim _{p \rightarrow \infty} w_{p}(x, y)=\lim _{p \rightarrow \infty} w_{k_{p}}(x, y),
$$

for any $(x, y)$ in $D$, it follows that

$4 \lim _{p \rightarrow \infty} w_{p}(m, n)=\lim _{p \rightarrow \infty} w_{p}(m+1, n)$

$$
+\lim _{p \rightarrow \infty} w_{p}(m-1, n)+\lim _{p \rightarrow \infty} w_{p}(m, n+1)+\lim _{p \rightarrow \infty} w_{p}(m, n-1),
$$

i.e., the limit function is a solution of the boundary value problem (notice that the existence of a solution has been proved independently of the earlier considerations involving Cramer's rule). The solution has already been shown to be unique by the maximum-minimum principle.

The restriction that the initial function $G$ be superharmonic will now be removed by showing that any function defined on $D+C$ may always be represented as the difference of two superharmonic functions on $D+C$. Let $g$ be any function defined on $D+C$, and let $M$ be one fourth of the maximum of the absolute value of the Laplacian of $g$ on $D$, i.e. $M=\frac{1}{4} \max _{D}|g(m+1, n)+g(m-1, n)+g(m, n+1)+g(m, n-1)-4 g(m, n)|$.

Then

$$
g(m, n)=g_{1}(m, n)+g_{2}(m, n)
$$

where

$$
\begin{aligned}
& g_{1}(m, n)=g(m, n)-M\left(m^{2}+n^{2}\right), \\
& g_{2}(m, n)=-M\left(m^{2}+n^{2}\right) .
\end{aligned}
$$

Clearly, $g_{1}$ and $g_{2}$ are both superharmonic on $D+C$. Thus, if $g$ is any function taking the prescribed values on $C$, the sequences of functions, constructed as explained above, corresponding to the initial superharmonic functions $g_{1}$ and $g_{2}$ converge to harmonic functions, and the difference of the two limit functions is the desired solution.

The method just described is the precise analogue of Poincarés [1] "method of sweeping out" for the classical Dirichlet problem. It also coincides with the relaxation method (Southwell [8]) provided that the relaxation is done point by point and that at each point the residual is actually reduced to zero. 


\section{Method II.}

This method differs from the preceding one only in the removal of the restriction that the process of method I be carried out pointwise at each step. Consider a sequence $B_{1}, B_{2}, B_{3}, \cdots$ of subregions ("blocks") of $D$, subject to the following conditions: (a) each point of $D$ is an interior point of an infinite number of the blocks of the sequence, (b) the Dirichlet problem is explicitly solvable for each block $B_{p}$ in terms of arbitrary boundary values on the boundary of $B_{p}$.

The convergence proof is the same as in method I. One need only substitute the sequence $B_{1}, B_{2}, B_{3}, \cdots$ of blocks for the sequence $P_{1}, P_{2}, P_{3}, \cdots$ of points.

This method is the exact analogue of the procedure suggested for the classical Dirichlet problem by Kellogg [2]. It is not to be confused with "block relaxation", with which it coincides only in the case where the residuals are actually made zero at each interior point of each block.

Method III.

Method III is essentially a method of successive approximations. Starting with an initial function $w_{0}=g$ which satisfies the prescribed boundary condition, one defines the following sequence of functions $w_{p}$ :

$$
\begin{aligned}
w_{0}= & g, \text { on } D+C ; \\
4 w_{1}(m, n)= & w_{0}(m+1, n)+w_{0}(m-1, n)+w_{0}(m, n+1) \\
& +w_{0}(m, n-1), \text { for }(m, n) \text { in } D, \\
w_{1}(m, n)= & g(m, n), \text { for }(m, n) \text { in } C ; \\
& \quad \cdot \\
4 & \quad+w_{p-1}(m, n-1), \text { for }(m, n) \text { in } D, \\
w_{p}(m, n)= & w_{p-1}(m+1, n)+w_{p-1}(m-1, n)+w_{p-1}(m, n+1) \\
w_{p}(m, n)= & g(m, n), \text { for }(m, n) \text { in } C ;
\end{aligned}
$$

Again one may suppose, without loss of generality, that the initial function $g$ is superharmonic to start out with. Hence all the functions $w_{p}$ are superharmonic and from the minimum principle for superharmonic functions it follows that

$$
w_{0} \geqq w_{1} \geqq w_{2} \geqq \cdots \geqq w_{p} \geqq \cdots \geqq \min _{c} g=\min _{c} f .
$$

Thus the sequence converges, and since

$$
\lim _{p \rightarrow \infty} w_{p}=\lim _{p \rightarrow \infty} w_{p-1},
$$


it follows that the limit function of the sequence is the desired solution of the Dirichlet problem.

The last method is related to the difference equation formulation of the heat flow problem, Emmons [9], in which the boundary $C$ is held at all times at a fixed temperature, $g$ represents the initial temperature, and the functions $w_{p}$ represent the temperature of $D+C$ at regular intervals of time after $t=0$. The limit function represents the steady state temperature. A proof of the convergence of method III, based upon random walk considerations, has been given by Feller [7].

\section{BiBLIOGRAPHY}

1. Poincaré, H., Amer. Journ. Math. 12, 211-294 (1890).

2. Kellogg, O. D., Foundations of potential theory, New York, 1941, specially p. 322.

3. Liebmann, H., Sitzungsber. Münchner Akad. 385-416 (1918).

4. Frank, P., and Mises, R. v., Differential und Integralgleichungen der Mechanik und Physik, vol. I, New York, 1943, specially p. 734.

5. Petrowsky, I. G., Lectures on partial differential equations, Moscow, 1950, specially p. 282.

6. Courant, R., Zeit. angew. Math. Mech. 6, 322-325 (1926).

7. Feller, W., and Tamarkin, J. D., Partial differential equations (mimeographed notes), Brown University, 1941, specially chapter $\mathrm{V}$.

8. Southwell, R. V., Relaxation methods in engineering science, Oxford, 1940.

9. Emmons, H. W., Quart. Appl. Math. 2, 173-195 (1944).

10. Moskovitz, D., Quart. Appl. Math. 2, 148-163 (1944). 